\title{
Compensation of position errors in passivity based teleoperation over packet switched communication networks
}

\author{
C. Secchi ${ }^{*}$ S. Stramigioli ${ }^{* *}$ C. Fantuzzi ${ }^{*}$ \\ * DISMI, University of Modena and Reggio Emilia, \\ via G. Amendola 2, Morselli Building, 42100 Reggio Emilia, Italy; \\ e-mail: \{cristian.secchi,cesare.fantuzzi\}@ unimore.it \\ ** EEMCS, University of Twente \\ P.O.Box 217, 7500 AE Enschede, NL \\ S.Stramigioli@ieee.org
}

\begin{abstract}
:
Because of the use of scattering based communication channels, passivity based telemanipulation systems can be subject to a steady state position error between master and slave robots. In this paper, we consider the case in which the passive master and slave sides communicate through a packet switched communication channel (e.g. Internet) and we provide a modification of the slave impedance controller for compensating the steady state position error arising in free motion because of packets loss.
\end{abstract}

\section{INTRODUCTION}

A bilateral telemanipulation system can be seen as a virtual mechanical coupling between two robots. The motion of the local robot, the master, has to influence that of the remote robot, the slave. On the other hand, the forces applied to the slave because of the interaction with a remote environment have to be propagated through the coupling to the master. This coupling is made up by the robots controllers and by the communication channel through which master and slave sides are interconnected. Ensuring the passivity of the overall telemanipulation system is sufficient for ensuring a stable behavior both in case of free motion and in case of contact with any passive environment. Passive impedance control has been used for ensuring the stability of the interactive behavior (see e.g. Lee and Li (2003); Itoh et al. (2000)) and the scattering framework (Anderson and Spong (1989); Niemeyer and Slotine (1991)) has been widely exploited for passivating the communication channel dynamics. In Stramigioli et al. (2002); Secchi et al. (2007), the port-Hamiltonian framework has been exploited for modeling and controlling passivity based bilateral telemanipulation systems. Master and slave are controlled through intrinsically passive port-Hamiltonian regulators which allow to shape the energetic behavior of the robots and to achieve desired dynamic properties at master and slave sides. Local and remote sides are interconnected through a scattering based communication channel that allows a lossless exchange of energy. The advantage of the port-Hamiltonian framework is that it allows to explicitly model the energetic structure of the systems and, therefore, it allows to keep track of the energy flows in the telemanipulation system. This can be very useful for the design of passive controllers. For a recent survey on bilateral teleoperation see Hokayem and Spong (2006).
When using scattering based communication channels, local and remote sides exchange only velocity and force information and this can cause the rise of a position error between master and slave robots as reported in Niemeyer (2004); Chopra et al. (2003); Lee and Spong (2005). Several works addressed the problem of position tracking in telemanipulation. In Chopra et al. (2003) an outer position loop is added to decrease the position error between master and slave; in Lee and Spong (2005) a novel control scheme for position regulation achieved passifying the control and the communication block is proposed. This problem has been recently tackled within the port-Hamiltonian framework. In Secchi et al. (2006), part of the energy dissipated by the port-Hamiltonian controller at the slave side has been stored and the interconnection structure of the controller has been modified in order to exploit the stored energy for compensating the steady state position error arising during contact tasks. In Stramigioli et al. (2005); Secchi et al. (2007) discrete scattering is used to build a control scheme for telemanipulation of port-Hamiltonian systems over a packet switched communication network (e.g. Internet). A strategy for passively dealing with the phenomena of packets loss has been proposed. Loosely speaking when an expected packet is not received, it is replaced by a null packets. Using this strategy, when a packet is lost, its corresponding energy content is dissipated. In this way, in free motion, some of the energy that has to be delivered to the slave side in order to move the slave robot accordingly with the master is lost and, therefore, at steady state, the position of the slave can be different from that of the master.

In this paper, we consider port-Hamiltonian based bilateral teleoperators as a quite general representation of passivity based bilateral telemanipulation systems. The portHamiltonian framework allows to model both linear and nonlinear passivity based telemanipulation systems. The 
goal of this work is to modify, in the same spirit as Secchi et al. (2006), the controller at the slave side for passively compensating the steady state position error due to the packets loss. Firstly we modify the controller, generalizing Secchi et al. (2006), in order to store ALL the energy that is dissipated by the controller and the amount of energy dissipated by the communication channel because of packets loss. Secondly, we modify the interconnection structure of the controller in order to use the energy that has been stored for passively compensating the steady state position error. Finally we put together the results obtained in this paper with those presented in Secchi et al. (2006) in order to obtain a controller that allows to passively compensate the steady state position errors that can arise both during contact tasks and in free motion.

\section{BACKGROUND}

Loosely speaking, a port-Hamiltonian system is made up of a set of energy processing elements (energy storing, energy dissipating and sources of energy) that exchange energy through a set of energy paths which form a power preserving interconnection along which energy is neither stored or dissipated but simply transferred. More formally, port-Hamiltonian systems are defined on the state manifold of energy variables $\mathcal{X}$ and they are characterized by an Hamiltonian energy function $H: \mathcal{X} \mapsto \mathbb{R}$, expressing the stored energy, and by a Dirac structure $\mathcal{D}$, representing the internal energetic interconnections. The system is endowed with a so-called power port that is represented by a pair of dual power variables $(e, f) \in V^{*} \times V$ called effort and flow respectively. This port is used to exchange energy with the system; the power supplied through a port is equal to $e^{T} f$. Using coordinates, in their simplest form, port-Hamiltonian systems are represented by the following equations

$$
\left\{\begin{array}{l}
\dot{x}(t)=(J(x)-R(x)) \frac{\partial H}{\partial x}+g(x) u(t) \\
y(t)=g^{T}(x) \frac{\partial H}{\partial x}
\end{array}\right.
$$

where $x \in \mathbb{R}^{n}$ is the coordinate vector of the energy variables $J(x)$ is a skew-symmetric matrix representing the Dirac structure, $R(x)$ is a positive semidefinite matrix representing the dissipation of the system, $H$ is the Hamiltonian function. The input-output pair $(u(t), y(t))$ forms the power port through which the system can exchange energy with the rest of the world. The system can either have an impedance causality or an admittance causality. In the first case $u(t)$ is a flow and $y(t)$ is an effort while in the second case $u(t)$ is an effort and $y(t)$ is a flow. The following power balance hold:

$$
y^{T}(t) u(t)=\dot{H}(t)+\frac{\partial^{T} H}{\partial x} R(x) \frac{\partial H}{\partial x}
$$

meaning that the energy provided through the port is either stored or dissipated and that a port-Hamiltonian system is a passive system. A very broad class of physical systems, both linear and non linear, can be modeled within the port-Hamiltonian framework which can therefore be used to model telemanipulation systems endowed with nonlinear robots. For further information, see for example Secchi et al. (2007).

The port-Hamiltonian based bilateral telemanipulation scheme is represented in Fig. 1 in a bond-graph notation.

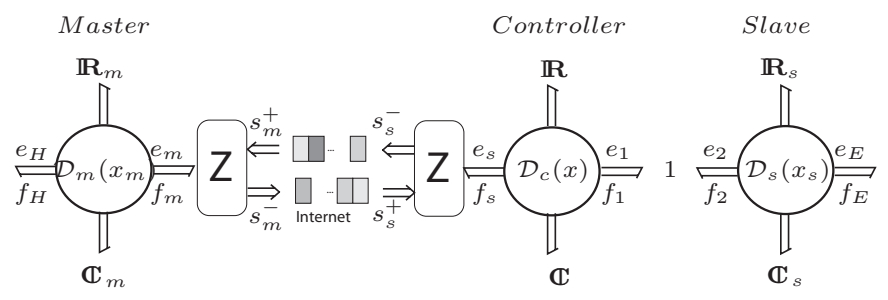

Fig. 1. The Port-Hamiltonian based Telemanipulation Scheme.

Both master and slave robots can be modeled as portHamiltonian systems. The slave is interconnected in a power preserving way to a port-Hamiltonian controller which acts as an intrinsically passive impedance controller. Master and slave sides exchange power through a transmission line that is characterized, in general, by a non negligible transmission delay. Each power port by means of which master and slave sides exchange power through the communication channel is characterized by an effort $e(t)$ and by a flow $f(t)$ and it can be equivalently represented by an incoming power wave $s^{+}(t)$ and an outgoing power wave $s^{-}(t)$ defined as

$$
\left\{\begin{array}{l}
s^{+}(t)=\frac{1}{\sqrt{2}} N^{-1}(e(t)+Z f(t)) \\
s^{-}(t)=\frac{1}{\sqrt{2}} N^{-1}(e(t)-Z f(t))
\end{array}\right.
$$

where $Z=N N>0$ is the symmetric positive definite impedance of the scattering transformation. The power flowing into the channel is given by $e^{T}(t) f(t)$ and it can be represented in terms of scattering variables as the difference between the incoming and the outgoing power flows, namely $e^{T}(t) f(t)=\frac{1}{2}\left\|s^{+}(t)\right\|^{2}-\frac{1}{2}\left\|s^{-}(t)\right\|^{2}$. In order to get a passive exchange of energy independently of any constant communication delay, the power ports connected to the transmission line are decomposed into a pair of scattering variables which are transmitted along the channel. Using this strategy, the communication channel is a lossless system and the power wave outgoing from the master side becomes the incoming power wave at the slave side and viceversa. Scattering variables can also be encoded into packets and transmitted through a packet switched transmission line while keeping on guaranteeing the losslessness of the communication channel. This kind of networks is unreliable and it can happen that some packets can be lost during the communication. In Stramigioli et al. (2005) a strategy for dealing with the loss of packets while preserving a passive behavior of the communication channel has been proposed: when a packet is not received either at the master or at the slave side, it is replaced with a null packet. Using this strategy, the loss of a packet corresponds to some energy dissipation and, therefore, the communication channel becomes a strictly passive system. The advantage of this strategy is that the communication channel keeps on being passive and that therefore no destabilizing effect is associated with packets loss. On the other hand, the energy associated to a lost packet is dissipated and never delivered. During free motion, this implies that the slave doesn't receive enough energy for reaching the same position of the master and, therefore, a steady state position error arises. 
Since a port-Hamiltonian based bilateral telemanipulator is made up of passive subsystems interconnected in a power preserving way, the overall system is intrinsically passive and, therefore, characterized by a stable behavior. For further details see Stramigioli et al. (2005); Secchi et al. (2007).

\section{STORAGE OF THE ENERGY DISSIPATED BY THE IMPENDANCE CONTROLLER}

The port-Hamiltonian impedance controller can be interpreted as a virtual physical system which usually contains an elastic term and some dissipative elements whose role is to impose a desired compliance and to dampen the interactive behavior of the slave. In this section we will show how it is possible to modify the slave controller for storing all the energy that it dissipates (and not only a part of it as proposed in Secchi et al. (2006)) and, furthermore, we will propose a communication strategy that allows also to store the energy that is dissipated by the packets loss phenomenon. This energy will then be used for compensating the position errors arising either in free motion or during contact tasks, as it will be explained in Sec. 4. The port-Hamiltonian impedance controller has usually an impedance causality and it can be modeled as

$$
\left\{\begin{array}{l}
\dot{x}(t)=(J(x)-R(x)) \frac{\partial H}{\partial x}+g(x)\left(\begin{array}{l}
f_{s}(t) \\
f_{1}(t)
\end{array}\right) \\
\left(\begin{array}{c}
e_{s}(t) \\
e_{1}(t)
\end{array}\right)=g^{T}(x) \frac{\partial H}{\partial x}
\end{array}\right.
$$

where $\left(e_{s}, f_{s}\right)$ and $\left(e_{1}, f_{1}\right)$ are the power ports through which the controller interacts with the communication channel and with the slave respectively.

In order to store the energy dissipated, it is necessary to endow the controller with an extra energy storing element that we call, as in Secchi et al. (2006), tank. A physical state has to be associated to this energy storage and, therefore, it is necessary to augment that state of the controller with an extra-energy variable that we call $x_{t} \in \mathbb{R}$. Furthermore, an energy function $H_{t}: \mathbb{R} \mapsto \mathbb{R}$ has to be associated to the tank and its role is to describe the amount of energy that is stored in correspondence of a given configuration of the tank $x_{t}$. The augmented model of the controller together with the tank where the energy dissipated is stored is given by

$$
\left\{\begin{array}{l}
\dot{x}(t)=(J(x)-R(x)) \frac{\partial H}{\partial x}+g(x)\left(\begin{array}{l}
f_{s}(t) \\
f_{1}(t)
\end{array}\right) \\
\dot{x}_{t}(t)=\frac{1}{\frac{\partial H_{t}}{\partial x_{t}}} \frac{\partial^{T} H}{\partial x} R(x) \frac{\partial H}{\partial x} \\
\left(\begin{array}{c}
e_{s}(t) \\
e_{1}(t)
\end{array}\right)=g^{T}(x) \frac{\partial H}{\partial x}
\end{array}\right.
$$

The energy stored in the tank is given by

$$
\int_{0}^{t} \dot{H}_{t}(\tau) d \tau=\int_{0}^{t} \frac{\partial H_{t}}{\partial x_{t}} \dot{x}_{t}(\tau) d \tau=\int_{0}^{t} \frac{\partial^{T} H}{\partial x} R(x(\tau)) \frac{\partial H}{\partial x} d \tau
$$

Namely, all the energy dissipated by the controller dynamics is stored in the tank. It is straightforward to see that augmented controller described in Eq.(5) is still passive with a storage function $H_{t o t}\left(x, x_{t}\right)=H(x)+H_{t}\left(x_{t}\right)$. In Eq.(5), we can see a singularity in case $\frac{\partial H_{t}}{\partial x_{t}}=0$. The Hamiltonian function of the tank $H_{t}$ has to be lower bounded in order to preserve the passivity of the controller but, apart of this condition, it can be freely chosen. A possible choice is $H_{t}\left(x_{t}\right)=\frac{1}{2} k x_{t}^{2}$. In this way $\frac{\partial H_{t}}{\partial x_{t}}=0$ only when $x_{t}=0$, namely when there is no energy stored in the tank. If we initialize the state of the tank with $x_{t}(0)>0$, namely if we put some initial energy into the tank, we have that $\frac{\partial H_{t}}{\partial x_{t}}(0)>0$. Using Eq.(5) and recalling that $R(x)$ is positive semidefinite, we can see that $x_{t}$ always increases and that $\frac{\partial H_{t}}{\partial x_{t}}$ will always be different form zero.

This implementation of the tank generalizes that proposed in Secchi et al. (2006) since all the dissipated by the controller is stored and the dimension of the state of the controller is augmented just by one. As reported in Sec. 2, when using the strategy proposed in Stramigioli et al. (2005), each time that a packet is lost in the communication, its energy content is dissipated. When using scattering variables, the communication channel becomes equivalent to a distributed mass spring system Niemeyer (2004) interconnecting the port-Hamiltonian master and slave sides. Thus, packets loss causes energy dissipation in this virtual distributed mechanical systems. As done for the controller we want to store the energy dissipated by the communication channel into the tank. The energy dissipated in the communication between master and slave sides and viceversa is given by the difference between the energy transmitted and the energy received, namely respectively by

$$
\begin{aligned}
& \Delta_{m s}(t)=\int_{0}^{t-T} \frac{1}{2}\left\|s_{m}^{-}(\tau)\right\|^{2} d \tau-\int_{0}^{t} \frac{1}{2}\left\|s_{s}^{+}(\tau)\right\|^{2} d \tau \\
& \Delta_{s m}(t)=\int_{0}^{t-T} \frac{1}{2}\left\|s_{s}^{-}(\tau)\right\|^{2} d \tau-\int_{0}^{t} \frac{1}{2}\left\|s_{m}^{+}(\tau)\right\|^{2} d \tau
\end{aligned}
$$

where $T$ denotes the communication delay and the indexes $m$ and $s$ stand for master and slave respectively. In case there is no packets loss, $s_{m}^{+}(t)=s_{s}^{-}(t-T)$ and $s_{s}^{+}(t)=$ $s_{m}^{-}(t-T)$ and, therefore, no energy is dissipated. In case of packets loss, some incoming packets are set to zero and the difference between the energy transmitted and the energy received is greater that zero and, therefore, a certain amount of energy has been dissipated. In order to be able to compute Eq.(7), it is necessary to embed in each transmitted packet both the value of the scattering wave $s_{i}^{-}(t)$ and the amount of energy that has been sent through the channel, $\int_{0}^{t} \frac{1}{2}\left\|s_{i}^{-}(\tau)\right\|^{2} d \tau$, where $i=m, s$. Thus each packet $P$ will have two fields $v$ and $e$ and

$$
\begin{aligned}
& \text { P.v }=s_{i}^{-} \\
& \text {P.e }=\int_{0}^{t} \frac{1}{2}\left\|s_{i}^{-}(\tau)\right\|^{2} d \tau
\end{aligned}
$$

At each side both the amount of energy transmitted through the channel and the amount of energy received from the channel are computed using local buffers. Let us consider the communication between master and slave. Each time that a packet $P$ is received, the amount of received energy $\int_{0}^{t} \frac{1}{2}\left\|s_{s}^{+}(\tau)\right\|^{2} d \tau$ is updated using P.v, the amount of energy transmitted by the master $\int_{0}^{t-T} \frac{1}{2}\left\|s_{s}^{+}(\tau)\right\|^{2} d \tau$ is read using P.e and $\Delta_{m s}$ is computed 
using Eq.(7). When a packet is not received, it is replaced with a packet where the $v$ field is set to zero, accordingly to Stramigioli et al. (2005), and the $e$ field is set at the same value of the corresponding field of the previously received packet. In this way $\Delta_{m s}$ remains unchanged. When the next packet $Q$ is received, the amount of energy received is updated using $Q . v$ and $\Delta_{m s}$ is computed using Q.e. We have that $\Delta_{m s}$ now increases since Q.e contains also the energy of the packets lost in the communication that have been replaced with null packets and that, therefore, haven't been considered in the computation of the energy received. The value of $\Delta_{m s}$ changes only in correspondence of some packets lost in the communication between master and slave. An analogous procedure at the master side allows to compute $\Delta_{s m}(t)$, namely the value of the energy dissipated in the communication between slave and master. Since the tank is embedded in the slave controller, it is necessary to communicate $\Delta_{s m}$ to the slave side. We can do this by adding a field, which we call $d$, to the packets transmitted from the master to the slave side and we set $P . d=\Delta_{m s}$. Thus, at the slave side, we can compute

$$
\Delta_{T O T}=\Delta_{m s}(t)+\Delta_{s m}(t-T)
$$

which represents all the energy that has been dissipated in the communication channel. Thus, we can store an amount of energy equal to $\Delta_{T O T}(t)$ in the tank. This can be done using the following tank dynamics

$$
\dot{x}_{t}(t)=\frac{1}{\frac{\partial H_{t}}{\partial x_{t}}} \frac{\partial^{T} H}{\partial x} R(x) \frac{\partial H}{\partial x}+\lambda f_{L}
$$

where

$$
\lambda=\left\{\begin{array}{c}
1 \text { if } \int_{0}^{t} f_{L}(\tau) \frac{\partial H_{t}}{\partial x_{t}} d \tau<\Delta_{T O T}(t) \\
0 \text { if } \int_{0}^{t} f_{L}(\tau) \frac{\partial H_{t}}{\partial x_{t}} d \tau=\Delta_{T O T}(t)
\end{array}\right.
$$

Using Eq.(10) we have that

$$
\dot{H}_{t}(t)=\frac{\partial^{T} H}{\partial x} R(x) \frac{\partial H}{\partial x}+\lambda \frac{\partial H_{t}}{\partial x_{t}} f_{L}
$$

Choosing $f_{L}>0$ and $H_{t}$ such that $\frac{\partial H_{t}}{\partial x_{t}}>0$ during the evolution of $x_{t}$ and using Eq.(11) we have that the second term of Eq.(12) injects energy into the tank as long as $\lambda>0$, namely as long as a total amount of energy equal to $\Delta$ has been stored into the tank.

\section{POSITION ERRORS COMPENSATION}

As already said, there are two possible steady state errors arising from the scattering based communication channel. The first one takes place during interaction tasks and it is due to the way in which the force feedback is passively transmitted to the user. The second one takes place during free motion and it is due to the loss of packets, namely to the fact that some of the required energy for performing a motion is not delivered. Both these kinds of position errors can be passively compensated by using the energy that is stored in the tank. The compensation of the steady state position error arising during contact tasks has been addressed in Secchi et al. (2006). The main idea is to model the rest length of one of the elastic elements that is present in the impedance controller as a state variable. It is then possible to create a controllable irreversible interconnection that allows to use some energy that is stored in the tank for passively changing the value of the rest length for introducing an offset that compensates the steady state position error.

In the same spirit, let us for example consider an elastic element that interconnects the slave robot with the scattering based communication channel (the results proposed in the paper can be easily extended also when considering other elastic elements connected to the slave). Let $x_{e}$ denote its state (which is part of the state of the controller) and let us model its rest length as an energy variable $x_{l} \in \mathbb{R}^{l}$, augmenting again the state of the controller; finally let $H_{e}\left(x_{e}-x_{l}\right)$ be the Hamiltonian function associated to this element. The model of this element can be described as

$$
\left\{\begin{array}{l}
\dot{x}_{e}(t)=f(t)=f_{s}(t)-f_{1}(t) \\
\dot{x}_{l}(t)=f_{l}(t) \\
e(t)=\frac{\partial H_{e}}{\partial x_{e}} \\
e_{l}(t)=\frac{\partial H_{e}}{\partial x_{l}}
\end{array}\right.
$$

where $(e(t), f(t))$ and $\left(e_{l}(t), f_{l}(t)\right)$ are the power ports through which the element exchange energy with the rest of the system. The effort $e(t)$ depends on the value of $x_{l}$ and is the one which is applied to the systems interconnected by the elastic element. Notice that it is possible to affect the value of $x_{l}$ through $f_{l}$ and that $\frac{\partial H_{e}}{\partial x_{e}}=-\frac{\partial H_{e}}{\partial x_{l}}$. A variation of $x_{l}$ affects the relative position of the systems interconnected by the elastic element.In case steady state position error arises in free motion, it is necessary to be careful in the error compensation process. In fact, if we changed the rest length of the elastic element for taking the slave to its correct steady state position, we would also apply a force to the distributed mass-spring system represented by the communication channel and this would have the effect of moving the master. This latter effect is undesired since we would like to change the slave position without requiring any correcting action of the user on the master and without transmitting to the master side any force due to the length variation. Another possible strategy would be to leave $x_{l}$ unchanged and to use the energy of the tank for applying an effort to the slave causing its motion. However, this would be unsuccessful since, because of the coupling between master and slave sides, a force applied to the slave would also cause an undesired motion of the master. The idea proposed in this paper is a mix of this two strategies: we create a controllable irreversible energy interconnection to drive some energy from the tank to the slave in order to move the slave and, while the slave is moving for reaching the master position, we change the rest length of the elastic element to compensate the force that would be transmitted to the master side because of the motion of the slave. First of all, let us build the interconnection that allows to transfer the energy from the tank to the slave. Let $\left(e_{t s}, f_{t s}\right) \in \mathbb{R} \times \mathbb{R}$ and $\left(e_{s c}, f_{s c}\right) \in \mathbb{R}^{m} \times \mathbb{R}^{m}$ the two power ports that are used for interconnecting the tank and the slave robot, the first one being referred to the tank and the second one to the slave. Let the interconnection between these two ports be given by:

$$
\left\{\begin{array}{l}
e_{s c}=B e_{t s} \\
f_{t s}=-B^{T} f_{s c}
\end{array}\right.
$$




$$
B=E e_{t s} f_{s c} \quad E=\operatorname{diag}\left(\varepsilon_{1}, \ldots \varepsilon_{m}\right)
$$

and $E$ is a design parameter. Using Eq.(14) we have that

$$
e_{s c}^{T} f_{s c}=e_{t s} B^{T} f_{s c}=-e_{t s} f_{t s}
$$

which means that the energy injected into the slave is equal to that extracted from the tank; thus Eq.(14) allows to transfer energy from the tank to the controller. Furthermore, we have that

$$
e_{s c}^{T} f_{s c}=e_{t s}^{2}\left(E f_{s c}\right)^{T} f_{s c}
$$

thus, if we choose $\varepsilon_{i}>0$ for $i=1, \ldots, m$ we inject energy into the slave and if we choose $\varepsilon_{i}<0$ for $i=1, \ldots, m$ we extract energy from the slave putting it back into the tank. Finally, setting $E=0$, we disable any energy transfer between the tank and the slave. Thus a proper choice of $E$ allows to control the energy flows between the slave and the tank. The magnitude of the gains $\varepsilon_{i}$ can be used to boost the energy transfer: the bigger are the magnitudes the faster is the energy transfer between the tank and the slave. By Eq.(14), we have that

$$
e_{s c}=E e_{t s}^{2} f_{s c}
$$

Suppose that the master position $q_{m}$ is embedded in the packet that is sent from master to slave side. If at steady state we have that the slave configuration $q_{s}(t)$ is different from $q_{m}(t-T)$, it means that some energy has not been delivered to the slave side. If $q_{s i}(t)<q_{m i}(t-T)$, it means that we have to apply to the slave a positive effort which takes $q_{s i}(t)$ to the correct position. This effort can be applied through the port $\left(e_{s c}, f_{s c}\right)$ using the energy stored in the tank. In order to decide whether to extract or to inject energy from/into the tank, we need to check the sign of the $i^{\text {th }}$ component of $f_{s c}$. If $f_{s c i}>0$ then we have to set $\varepsilon_{i}>0$ else, we have to set $\varepsilon_{i}<0$. A similar reasoning holds in case $q_{s i}(t)<q_{m i}(t-T)$ and an effort with the $i^{\text {th }}$ component negative has to be applied. By repeating these steps for all the components of $q_{s}(t)$ it is possible to determine $E$ such that it allows to transfer from the tank an amount of energy that allows to take the slave at the master configuration, compensating the steady state position error. The sign of the elements $\varepsilon_{i}$ determines the direction of the energy transfer while their value acts as a gain which boosts the energy transfer. The greater are the values of the gains, the faster is the energy transfers and, consequently, the transient of the compensation process. Thus, properly tuning of the energy transfer between the tank and the length port, it is possible to achieve any desired transient behavior in the rest length variation. As explained above, the motion of the slave as an effect of the application of the effort $e_{s c}$ hasn't to affect the master position. At steady state, the effort applied by the elastic element described in Eq.(13) is $e=0$. Thus both the effort applied by the element to the slave and to the scattering channel is zero. We would like to preserve this situation also during the compensation phase, namely when the slave starts extracting energy from the tank for changing its position. Suppose, for simplicity, that $H_{e}$ has just a global minimum and that $\operatorname{argmin} H_{e}=0$. The results proposed in the following can be easily extended to more general cases. Thus, at steady state, $x-x_{l}=0$. Using Eq.(13) we have that

$$
x(t)=\int_{0}^{t} f_{s}(\tau) d \tau-\int_{0}^{t} f_{1}(\tau) d \tau=\tilde{q}_{m}(t)-q_{s}(t)
$$

At steady state we have that

$$
\tilde{q}_{m}(t)-q_{s}(t)-x_{l}(t)=0
$$

If we can guarantee that Eq.(20) keeps on holding during the compensation process, we have that the effort transmitted by the elastic element to the master keeps on being zero and that, therefore, the motion of the slave doesn't affect that of the master. During the compensation process $\dot{q}_{s}(t)=f_{s}(t) \neq 0$ and, trivially, if we set

$$
\dot{x}_{l}=-\delta f_{s}(t)
$$

where

$$
\delta= \begin{cases}1 & E \neq 0 \\ 0 & E=0\end{cases}
$$

it can be immediately seen that Eq.(20) keeps on holding during the compensation phase and that, therefore, no effort is sent to the master side and the compensation process is transparent to the user.

When changing $x_{l}$, we have that $e=\frac{\partial H_{e}}{\partial x_{e}}=-\frac{\partial H_{e}}{\partial x_{l}}=e_{l}=$ 0 . Thus we have that $e_{l}^{T} f_{l}=0$ and, therefore, no energy is required for the length variation process.

\section{THE OVERALL CONTROLLER}

Summarizing the results obtained in the previous sections and the result proposed in Secchi et al. (2006), we obtain the following controller:

$$
\left\{\begin{array}{l}
\dot{x}=[J(x)-R(x)] \frac{\partial H}{\partial x}+g(x)\left(\begin{array}{l}
f_{s} \\
f_{1}
\end{array}\right) \\
\dot{x}_{t}(t)=\frac{1}{\frac{\partial H_{t}}{\partial x_{t}}} \frac{\partial^{T} H}{\partial x} R(x) \frac{\partial H}{\partial x}+A \frac{\partial H}{\partial x_{l}}+B^{T} f_{1}(t)+\lambda f_{L} \\
\dot{x}_{l}=-A^{T} \frac{\partial H_{t}}{\partial x_{t}}-\delta f_{1}(t) \\
\left(\begin{array}{c}
e_{s} \\
e_{1}
\end{array}\right)=g^{T}(x) \frac{\partial H}{\partial x}+\left(\begin{array}{c}
0 \\
B \frac{\partial H_{t}}{\partial x_{t}}
\end{array}\right)
\end{array}\right.
$$

The dynamics of the original set of state variables $x$ remains that of the port-Hamiltonian impedance controller initially designed for the telemanipulation system. Thus the user, when the compensation processes are disabled, feels exactly the desired impedance. The state variables $x_{t}$ and $x_{l}$ represent the tank and the rest length dynamics respectively. The coupling modeled by matrix $A$ allows to exploit the energy of the tank for changing $x_{l}$ in order to compensate the position errors arising during contact tasks Secchi et al. (2006). We can easily prove the following Proposition 1. Using the controller proposed in Eq.(23) in the port-Hamiltonian based telemanipulation scheme reported in Sec. 2, leads to a passive telemanipulation system.

Proof. The total energy of the controller is given by $H_{T O T}=H\left(x, x_{l}\right)+H_{t}\left(x_{t}\right)$. Using Eq. (23) and exploiting the skew-symmetry of $J(x)$, we have that

$$
\begin{aligned}
\dot{H}_{T O T}=\frac{\partial^{T} H}{\partial x} g(x)\left(\begin{array}{c}
f_{s} \\
f_{1}
\end{array}\right)-\delta \frac{\partial^{T} H}{\partial x_{l}} f_{1} & +\frac{\partial^{T} H_{t}}{\partial x_{t}} B^{T} f_{1}+ \\
& +\lambda \frac{\partial^{T} H_{t}}{\partial x_{t}} f_{L}
\end{aligned}
$$

Consider the second term. If $\delta \neq 0$, it means that the slave is moving for compensating the steady state position error and, therefore, as reported in Sec. 4, we have that 


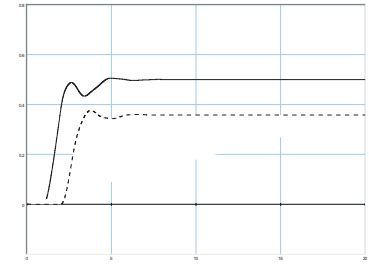

(a) no compensation

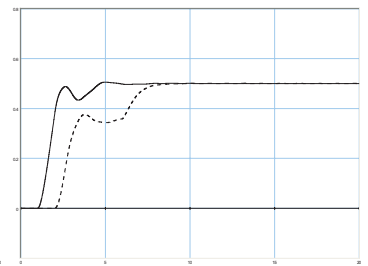

(b) compensation
Fig. 2. Master(solid) and Slave (dashed) Positions

$\frac{\partial H}{\partial x_{l}}=0$. Thus, this term is always equal to zero. Using the last equation of Eq.(23) we get

$$
\dot{H}_{T O T}=e_{s}^{T} f_{s}+e_{1}^{T} f_{1}+\lambda \frac{\partial H_{t}}{\partial x_{t}} f_{L}
$$

By construction, the last term is always greater or equal to zero and this implies that the controller produces energy and that, therefore, it is not passive. Nevertheless, as reported in Sec. 3, the amount of energy produced by the controller is exactly equal to that dissipated by the communication channel and, therefore, the system made up of the controller and of the communication channel is lossless and, therefore, passive. Thus, the coupling between master and slave is passive. Furthermore, since the lossless system given by the controller and by the communication channel is interconnected to two passive systems, the robots, in a power preserving way and since the power preserving interconnections of passive systems yields a passive system Secchi et al. (2007), we have that the overall telemanipulation system is passive.

\section{SIMULATIONS}

We consider a simple one degree of freedom telemanipulator where master and slave are simple masses of $0.5 \mathrm{Kg}$. The slave is interconnected to a port-Hamiltonian impedance controller and local and remote sides are joined through a scattering based communication channel characterized by an impedance $Z=1$ and by a transmission delay $T=0.5 \mathrm{~s}$ in both senses of communication. The portHamiltonian controller is a simple PD, physically equivalent to the parallel of a spring with stiffness $K=10 \mathrm{~N} / \mathrm{m}$ and a damper with dissipation coefficient $b=1 \mathrm{~N} \mathrm{sec} / \mathrm{m}$ used to match the impedance of the communication channel. The tank is an energy storing element with $H_{t}=\frac{1}{2} x_{t}^{2}$ and it is initialized with $x_{t}(0)=1$ in order to avoid the singularity problems mentioned in Sec. 3. We have simulated the behavior. In the simulation, the user is applying an force pulse at the master and, in the communication between master and slave, the sequence of packets that has to be received at the slave side in $t \in[1,2]$ is is lost and replaced with a sequence of null packet accordingly with Stramigioli et al. (2005). The results of the simulation have been reported in Fig. 2. We can notice from Fig. 2(a) that the telemanipulation system remains stable despite of the loss of information in the communication since the overall system keeps on being passive. Nevertheless, the fact that some of the energy that should have been delivered to the slave is dissipated causes a significant steady state position error. In Fig. 2(b) the positions of master and slave are reported in case the compensation algorithm reported in Sec. 4 has been implemented. We can see that when the slave reaches the steady state position and a significant difference between the slave and and the master position is detected, part of the energy of the tank is used for moving the slave towards the correct configuration and, at the same time, the rest length of the spring in the PD controller is changed as explained in Sec. 4 to avoid that the corrective motion of the slave affects also the master. In fact, we can see that the steady state position of the master remains unchanged after the compensation phase. Thus, at the end, both master and slave positions are equal to the value set by the user.

\section{CONCLUSIONS AND FUTURE WORK}

In this paper we have proposed an control strategy for passively compensating the steady state position error arising in free motion when some packets are lost in the communication between master and slave sides.In the future, we aim at exploiting the control structure proposed in Sec. 5 for implementing corrective actions to improve the transparency of the telemanipulation system also during the motion of the slave.

\section{REFERENCES}

R. Anderson and M. Spong. Bilateral control of teleoperators with time delay. IEEE Trans. on Automatic Control, 34(5):494-501, 1989.

N. Chopra, M.W. Spong, S. Hirche, and M. Buss. Bilateral teleoperation over the internet: the time varying delay problem. In Proc. of American Control Conference, volume 1, Denver, Colorado, USA, June 2003.

P.F. Hokayem and M.W. Spong. Bilateral teleoperation: An historical survey. Automatica, 42(12), 2006.

T. Itoh, K. Kosuge, and T. Fukuda. Human-machine cooperative telemanipulation wit motion and force scaling using task-oriented virtual tool dynamics. IEEE Transactions on Robotics and Automation, 16(5), 2000.

D. Lee and P.I. Li. Passive bilateral feedforward control of linear dynamically similar teleoperated manipulators. IEEE Trans. on Robotics and Automation, 19(3):443456, 2003.

D. Lee and M.W. Spong. Passive bilateral control of teleoperators under constant time-delay. In Proc. of IFAC world Congress, Prague, July 2005.

G. Niemeyer and J. Slotine. Stable adaptive teleoperation. IEEE Journal of Oceanic Engineering, 16(1):152-162, 1991.

J.-J. Niemeyer, G. ans Slotine. Telemanipulation with Time Delays. International Journal of Robotics Research, 23(9):873-890, September 2004.

C. Secchi, S. Stramigioli, and C. Fantuzzi. Position drift compensation in port-hamiltonian based telemanipulation. In Proc. of IEEE/RSJ Int. Conf. on Intelligent Robots and Systems, Beijing, China, 2006.

C. Secchi, S. Stramigioli, and C. Fantuzzi. Control of Interactive Roboti Interfaces: a port-Hamiltonian Approach. Springer Tracts in Advanced Robotics. Springer, 2007.

S. Stramigioli, C. Secchi, A.J. van der Schaft, and C. Fantuzzi. Sampled data systems passivity and discrete porthamiltonian systems. IEEE Trans. on Robotics, 21(4): 574-587, 2005.

S. Stramigioli, A. van der Schaft, B. Maschke, and C. Melchiorri. Geometric scattering in robotic telemanipulation. IEEE Trans. on Robotics and Automation, 18(4), 2002. 